\title{
El Sistema Nacional de Investigadores en México: 20 años de producción científica en las instituciones de educación superior (1991-2011)
}

\author{
Jorge Rodríguez Miramontes* \\ Claudia N. González Brambila** \\ Gabriela Maqueda Rodríguez*
}

Artículo recibido:

7 de marzo de 2016

Artículo aceptado:

27 de septiembre de 2016

\section{RESUMEN}

El objetivo de este trabajo es analizar cómo un sistema de estímulos económicos y de reconocimiento a las actividades de investigación en México ha impactado la productividad científica de las instituciones de educación superior (IES) a nivel nacional. Este análisis emplea una base de datos con información de 27,667 investigadores mexicanos que han formado parte del Sistema Nacional de Investigadores (SNI), al menos por un año, en el periodo 1991-2011, e integra 122,406 artículos publicados por dichos científicos en revistas

DCTS-Centro de Investigación y Estudios Avanzados (Cinvestav), Instituto Politécnico Nacional (IPN), México. jrodriguezm@cinvestav.mx, gmaqueda@cinvestav. $\mathrm{mx}$.

**Instituto Tecnológico Autónomo de México (ITAM), México. cgonzalez@itam.mx.

INVESTIGACIÓN BIBLIOTECOLÓGICA, Número Especial de Bibliometría, 2017, México, ISSN: 2448-8321. pp 187-219 
ISI. A fin de determinar la cantidad y calidad de la productividad de las IES, se consideraron como unidades de medida los artículos de publicaciones científicas y las citas bibliográficas. Los resultados indican que únicamente el $28 \%$ de las IES analizadas emitieron uno o más artículos en el periodo de estudio. Adicionalmente, nuestros resultados muestran que en diez universidades se agrupa tanto la mayor cantidad de artículos publicados, como la mayor cantidad de citas bibliográficas. Por otra parte, se muestra que la edad máxima de productividad es a los 58 años para el caso de las investigadoras y 57 años para los investigadores. Finalmente, una de las contribuciones de este trabajo es que muestra que las actividades de colaboración están en continuo crecimiento entre los investigadores.

Palabras clave: SNI; IES; Producción científica; Colaboración.

The National Researchers System in Mexico: 21 years of research output at institutions of higher education (1991-2011)

Jorge Rodríguez Miramontes, Claudia N. González Brambila and Gabriela Maqueda Rodríguez

\section{Abstract}

The aim of this paper is to analyze how a system of economic stimulus and recognition to the research activities in Mexico has impacted the scientific productivity of Higher Education Institutions (HEI). This analysis uses a database with information of 27,667 Mexican researchers who have been benefited from the Sistema Nacional de Investigadores (SNI) at least for one year among the period 1991-2011 and includes 122,406 articles published by these researchers in ISI journals. In order to determine the quantity and quality of the productivity of HEI we considered as units of measurement the publications and citations. The results indicate that only $28 \%$ of the HEI analyzed 
issued one or more publications in the study period. Additionally, our results show that in 10 universities are grouped both the higher quantity of publications and the higher quantity of quotes. Moreover, it is shown that the maximum age of productivity is at 58 years in the case of the female researchers and 57 years for male researchers. Finally, one of the main contributions of this work is that it show that collaboration activities are continuously growing among researchers.

Keywords: SNI, HEI; Research output; Collaboration.

\section{INTRODUCCIÓN}

T a evaluación de las actividades de investigación se basa en un proceso Lde recolección de información, cuyo objetivo es asegurar la calidad de la investigación y de la educación, mediante el funcionamiento eficiente de las instituciones de educación superior (IES). La importancia y frecuencia de la evaluación, así como el monitoreo de los resultados de la investigación científica y tecnológica ha incrementado desde los años ochenta a escala mundial. A nivel macro, los gobiernos han optado por aumentar el financiamiento a los proyectos, en detrimento del financiamiento institucional. A fin contar con recursos económicos, los investigadores someten propuestas de trabajo en instituciones financiadoras, las cuales comúnmente emplean evaluadores externos e internos para seleccionar las propuestas que serán beneficiadas.

Existe evidencia que muestra que el número de artículos publicados por parte del investigador postulante tiene un efecto considerable en las decisiones de los evaluadores a la hora de determinar quiénes recibirán financiamiento. A nivel micro, las universidades y centros de investigación igualmente consideran el número de artículos y citas bibliográficas para supervisar el desempeño de los investigadores, así como para otorgar compensaciones, promociones y ascensos.

En el ámbito del investigador, esta dinámica ha propiciado dos fenómenos: "Publicar o perecer" y "Publicar o enseñar". El término "Publicar o perecer" describe la presión de los investigadores por publicar rápida y continuamente, a fin de mantener un estatus y permanecer en un grupo selecto dentro de la academia; mientras que la expresión "publicar o enseñar" se refiere al doble papel que desempeñan los profesores dentro de una universidad: investigar e impartir cátedra. En ambos casos, la presión se incrementa cuando la contratación, la permanencia o la promoción dependen de los 
resultados de cada investigador (artículos publicados y citas bibliográficas). Sin embargo, cabe considerar que la productividad de los científicos también se asocia con el apoyo con que cuentan para desarrollar con éxito sus tareas y que dicho apoyo se encuentra estrechamente relacionado con el lugar de adscripción de cada uno de ellos.

El objetivo de este trabajo es analizar cómo un sistema de estímulos económicos y de reconocimiento a las actividades de investigación en México ha impactado en la productividad científica de las IES a escala nacional. Una de las principales contribuciones de este trabajo es que se realiza un estudio cienciométrico, a partir de una base de datos integrada por información procedente de un concurso entre la base de datos del Science and Social Sciences Citation Index, desarrollados por el Institute of Scientific Information (ISI, 2012) y la base de datos de los investigadores que han sido parte del SNI de México.

\section{CONSIDERACIONES TEÓRICAS}

A continuación, se describen algunos de los factores que inciden en la productividad de los investigadores (número de artículos publicados y número de citas bibliográficas).

\section{La educación superior en México}

En México y en América Latina, las organizaciones académicas se clasifican en dos tipos: cátedra-facultad y departamento-colegio. La primera está integrada por unidades operacionales dentro de una universidad, es decir, son células enfocadas en un plan de estudio (carrera), las cuales agrupan a estudiantes inscritos en un programa y a profesores que, independientemente de su disciplina, están adscritos a la división o carrera de estudio. Dicha organización por lo general está presidida por un director de división o carrera.

Por otra parte, la organización departamento-colegio se distingue por el agrupamiento de profesores e investigadores en un departamento, quienes se especializan en un área determinada del conocimiento y que conjuga las actividades de investigación y de docencia en una sola unidad académica.

El surgimiento de la universidad moderna (caracterizada por su dedicación a la investigación), ocasionó que el modelo catedra-facultad entrara en crisis. Acorde a lo documentado por Alcántara (2009), este modelo latinoamericano entró en declive a finales de los setenta y principios de los ochenta, debido a motivos como el incremento de la matrícula y la diversificación de las IES en los países de la región, la percepción del fracaso público, el surgimiento de la economía del conocimiento y el capitalismo académico, principalmente. 
En México, ejemplo de la transformación del modelo clásico (catedra-colegio) al modelo moderno (departamento-colegio), se presentó a principios de los setenta, en la Universidad Autónoma de México (UNAM), al diferenciar las entidades dedicadas a la docencia (facultades y escuelas) de las entidades cuyo objetivo principal es la investigación (institutos y centros), buscando una sinergia entre ambas funciones. Bajo el nombre de "universidad de investigación" o "universidad moderna", esta sinergia se caracteriza por una organización departamental, con un sistema de jerarquización y promoción de los profesores, burocracia profesional y eficiente, flexibilidad curricular, un gobierno que está en manos de los académicos, así como por un sistema de reconocimientos y estímulos económicos para la investigación.

En los años setenta, el modelo adoptado por la UNAM también fue acogido por otras IES privadas, como el Instituto Tecnológico de Estudios Superiores Monterrey (ITESM) y la Universidad Autónoma de Guadalajara (UAG), incluso en 1974 se creó la Universidad Autónoma Metropolitana (UAM), con un enfoque totalmente de departamento-colegio. Sin embargo, debido las condiciones académicas y financieras, en México aún persisten las IES con un enfoque más orientado a la docencia que a la investigación.

\section{El Sistema Nacional de Investigadores (SNI)}

La relación entre el salario y la productividad del investigador se ha documentado en la literatura desde hace varios años. Principalmente se ha demostrado que cuando una IES otorga salarios competitivos y atractivos, se crea una atmósfera de seguridad y satisfacción por parte de los investigadores, lo que influye positivamente en la generación de actividades científicas de alta calidad, y a su vez disminuye el riesgo de "fuga de cerebros" hacia otras IES. Sin embargo, no todas las universidades en México pueden ofrecer salarios atractivos a sus docentes, lo que implica un problema para los investigadores, quienes deben demostrar ser productivos a fin de asegurar su permanencia y promoción en las IES.

Para atender la problemática relacionada con su salario, los investigadores han optado por obtener recursos extras (becas) provenientes de diversos programas. Se ha documentado que, en algunos casos, el 50\% de los ingresos que perciben los académicos corresponden a recursos externos. De acuerdo a lo descrito por Merton (1957), la ciencia ha desarrollado un sistema de recompensas diseñado para dar "el reconocimiento y estima a los científicos que han cumplido mejor sus funciones, a los que han hecho contribuciones genuinamente originales al conocimiento". 
En respuesta a la situación que enfrentaba la comunidad científica en México, a raíz de la crisis de 1982, por decreto del presidente Miguel de la Madrid, el 26 de julio de 1984 se creó el Sistema Nacional de Investigadores (SNI), sistema instaurado para reconocer la labor de las personas dedicadas a generar conocimiento y, a su vez, representó un mecanismo para promover y fortalecer (a través de la evaluación) la calidad de la investigación científica y tecnológica que se produce en el país.

El reconocimiento a la labor de investigación se otorga por medio de la evaluación por parte de comisiones dictaminadoras integradas por pares, las cuales corresponden a cada una de las áreas del conocimiento. El objetivo de estas comisiones es evaluar la calidad académica, la trascendencia y el impacto de la investigación científica y tecnológica, así como la docencia y la formación de recursos humanos por parte de los investigadores. Básicamente, los productos de la investigación científica y tecnológica son artículos, libros, patentes, desarrollos tecnológicos, innovaciones y transferencias tecnológicas; mientras que la docencia y la formación de recursos humanos corresponde a la dirección de tesis, impartición de cursos, así como a la formación de investigadores y de grupos de investigación.

El reconocimiento o la distinción que otorga el SNI representa la calidad de las contribuciones realizadas por el científico al fortalecimiento de la investigación en el país. La distinción otorgada a los investigadores se clasifica en tres categorías: 1) candidato a investigador nacional; 2) investigador nacional (con tres niveles) y 3 ) investigador nacional emérito. Aunado a la distinción, el SNI puede otorgar estímulos económicos cuyo monto oscila entre cuatro y quince salarios mínimos mensuales (exentos de impuestos). Sin embargo, para recibir dicho estímulo económico, el investigador debe de tener un contrato en una IES. Cabe mencionar que, tanto la distinción como el estímulo económico, no son permanentes (salvo la distinción de investigador emérito nacional, la cual es vitalicia) y que la vigencia depende del tipo de categoría: candidato $=$ validez de tres años (con derecho a prórroga de dos más); investigador nacional nivel $1=$ validez de tres años; cuatro años de validez para el nivel 2 y de cinco años para el nivel 3.

\section{Publicar y enseñar o salir del SNI}

Acorde a lo descrito por Craswell (1986), la productividad científica es el grado en el que los académicos se comprometen con su propia investigación, mediante la publicación de artículos científicos en revistas indexadas, conferencias en congresos, publicación de libros, formación de recursos humanos, obtención de patentes, licenciamientos y financiamiento, entre otras 
actividades. Básicamente, la productividad científica se describe como un proceso de insumo-producto (input-output); los insumos consisten en recursos financieros y humanos (financiamiento, becas, salarios de investigadores, entre otros) y los productos son todos los resultados derivados del proceso de la investigación de un académico.

En general, los productos se clasifican como tangibles (patentes, artículos, libros, presentaciones en congresos y demás actividades) e intangibles (consultoría, madurez del conocimiento, entre otros). Sin embargo, se consideran tres parámetros clásicos empleados para medir el desempeño de un investigador o de una IES: conteo de artículos en publicaciones científicas, conteo de citas bibliográficas y clasificación de pares o universidades; siendo el número de artículos en revistas académicas específicas la medida más empleada para evaluar la productividad de un investigador.

La mecánica antes descrita ha propiciado entre los investigadores la presión por publicar rápida y constantemente, lo que deriva en prácticas indeseables, como estudios triviales para obtener resultados rápidos, reportar estudios más de una vez, fraudes e inclusión de autores que no contribuyeron en la investigación, entre otros.

Para los científicos, el SNI es sinónimo de prestigio, pues representa un círculo selecto que distingue a los investigadores que efectúan con mayor eficiencia su trabajo y realizan contribuciones importantes al conocimiento; en tanto que, desde el punto de vista económico, dicho sistema implica un "complemento salarial". Conforme a lo descrito por Maldonado (2010), en México los salarios de los profesores en las IES en 2009 oscilaban entre $\$ 4,800$-\$31,000 en instituciones públicas; mientras que en las instituciones privadas el monto fue de $\$ 5,276-\$ 54,104$. El salario de los investigadores con categoría de profesor investigador de tiempo completo oscilaba entre \$11,971-\$21,356 en el sector público; en cambio, en el privado oscilaba entre $\$ 13,325-\$ 27,764$, en promedio.

Si se considera que en 2009 el salario mínimo fue $\$ 54.8$ y que el monto del estímulo que otorga el SNI en forma de beca ronda entre cuatro y quince salarios mínimos mensuales, el monto del apoyo recibido por parte de los investigadores adscritos al SNI osciló entre \$6,576-\$24,660 mensuales, lo que corresponde a un ingreso extra del $54-200 \%$ para los científicos con categoría más baja y para la categoría más alta el ingreso extra representó 30-115\% de su salario mensual.

Si un investigador desea incrementar sus ingresos, lo puede hacer de manera institucional con una recategorización, o mediante el sin, con el estímulo económico. En ambos casos, el científico se debe someter a una evaluación por pares, para determinar la cantidad y calidad de sus contribuciones al 
conocimiento, lo cual permitirá establecer su categoría en la IES de adscripción o su nivel en el SNI.

Cabe señalar aquí que la frecuencia con que se emiten las convocatorias para promoción en las IES depende de los recursos económicos autorizados de la institución para ese fin (en algunas pueden pasar años, lo que ocasiona que el investigador no avance en el tabulador), mientras que en el SNI la convocatoria se emite anualmente, lo cual permite al académico escalar en menor tiempo los niveles del sistema e incrementar sus ingresos.

La presión por publicar inicia desde que el científico busca ingresar al SNI y continúa cuando se desea asegurar la permanencia o, en su momento, cuando solicita una reincorporación. La presión depende de la cantidad de artículos publicados necesarios para ingresar por nivel, la cual varía en cada área del conocimiento, como se describe en el Cuadro 1.

En algunos casos, se tiene la certeza del número mínimo de artículos; sin embargo, en ciertas áreas del conocimiento la cantidad depende de la evaluación de la comisión dictaminadora. En la mayoría de las áreas se ingresa al nivel más bajo, con una publicación como autor principal, y para mantenerse o avanzar a otros niveles, se requieren cinco artículos en un promedio de tres años.

Cuadro 1. Artículos mínimos para ingreso al sNI por área

\begin{tabular}{|l|l|l|l|l|}
\hline \multirow{2}{*}{\multicolumn{1}{|c|}{ Área }} & \multicolumn{1}{|c|}{ Candidato } & \multicolumn{1}{c|}{ I } & \multicolumn{1}{c|}{ II } & \multicolumn{1}{c|}{ III } \\
\cline { 2 - 5 } & No especificado & No especificado & No especificado & No especificado \\
\hline $\begin{array}{l}\text { I. Físico-Matemá- } \\
\text { ticas y Ciencias de } \\
\text { la Tierra }\end{array}$ & $\begin{array}{l}2 \text { (autor principal) } \\
0203 \text { (coautor) }\end{array}$ & $\begin{array}{l}1 \text { por año (prome- } \\
\text { dio) }\end{array}$ & $\begin{array}{l}15-25 \\
4 \text { en 3 años }\end{array}$ & $\begin{array}{l}15-25 \text { (con } \\
\text { citas bibliográficas } \\
\text { promedio) }\end{array}$ \\
\hline $\begin{array}{l}\text { II. Biología y } \\
\text { Química }\end{array}$ & No especificado & No especificado & No especificado & No especificado \\
\hline $\begin{array}{l}\text { III. Medicinay } \\
\text { Ciencias de la } \\
\text { Salud }\end{array}$ & $\begin{array}{l}1 \text { (autor principal) } \\
0 \text { más de } 1 \text { (coau- } \\
\text { tor) }\end{array}$ & 5 & No especificado & No especificado \\
\hline $\begin{array}{l}\text { IV. Humanidades } \\
\text { y Ciencias de la } \\
\text { Conducta }\end{array}$ & $\begin{array}{l}1 \text { (autor principal) } \\
02 \text { (coautor) }\end{array}$ & 5 & 5 & 5 \\
\hline $\begin{array}{l}\text { V. Ciencias } \\
\text { Sociales }\end{array}$ & 1 (autor principal) & No especificado & No especificado & No especificado \\
\hline $\begin{array}{l}\text { VI. Biotecnologíay } \\
\text { Ciencias Agrope- } \\
\text { cuarias }\end{array}$ & No especificado & No especificado & No especificado & No especificado \\
\hline \begin{tabular}{l} 
VII. Ingenierías \\
\hline
\end{tabular}
\end{tabular}

Fuente: elaboración propia, con datos del Conacyt (2015). 
Uno de principales problemas a los que se enfrentan los profesores en una universidad es administrar su tiempo, a fin de desempeñar dos actividades de manera simultánea: investigar y enseñar. Como observan Melguizo y Strober (2007), los investigadores son recompensados económicamente por incrementar su productividad, con lo cual aumentan su prestigio (y el de la universidad), lo que redunda en que los profesores dediquen más tiempo a estas actividades que a las de docencia (la enseñanza, asesorías y tutoría), debido a que éstas no tienen un gran peso para decisiones relacionadas con el incremento salarial de los investigadores.

FACTORES INCIDENTES EN LA PRODUCTIVIDAD DE LOS INVESTIGADORES

\section{Género}

En la literatura se han documentado diversos estudios acerca de los determinantes de la productividad, siendo la variable género uno de los factores más analizados. Empleando una muestra de 262 sociólogos, Babchuk y Bates (1962) documentaron una tasa más baja de productividad de las mujeres respecto de los hombres.

Posteriormente, mediante una encuesta del American Council on Education, Astin (1972) indicó que el 26\% de las mujeres nunca habían publicado en una revista académica especializada, mientras que el porcentaje respecto de los hombres disminuía al 10\%. Posteriormente, Cole y Zuckerman (1984) realizaron un estudio con 256 científicos y documentaron que, en promedio, los investigadores publican entre un 40 a 50\% más artículos que las investigadoras.

Por su parte, Kyvik (1990) empleó una encuesta aplicada a investigadores en la Comunidad Económica Europea, la cual fue realizada por Franklin (1989); mediante su análisis encontró que las investigadoras publican en promedio cinco artículos en un periodo de tres años, en contraste con ocho artículos que publican los investigadores en el mismo periodo. Stack (2004), mediante datos obtenidos en 1995 en una encuesta aplicada a 11,231 investigadores en ciencias e ingeniería, documentó que las mujeres publican significativamente menos en relación con los hombres, e indicó que en el área de las ciencias sociales (en las que existe una mayor concentración de mujeres), el género no está relacionado con la productividad, aunque las mujeres con hijos jóvenes tienen una producción relativamente baja. 


\section{Edad}

La relación que existe entre la edad y la productividad de los científicos es un tema que se ha documentado plenamente. Un estudio pionero fue el de Lehman (1953), quien documentó que los principales hallazgos de los investigadores los realizan entre los 30 y 40 años, lo cual sugiere que la edad se correlaciona negativamente con la productividad.

Por su parte, Pelz y Andrews (1966), empleando datos de 1,300 científicos e ingenieros, identificaron que la productividad máxima se presentó entre los 35-44 años, y posteriormente entre los 50-54 años de los investigadores. Mediante una muestra representativa de científicos académicos en Estados Unidos, en siete campos de la ciencia, Bayer y Dutton (1977) documentaron que en cinco de los siete casos analizados se presentan dos máximos en relación con la productividad de los investigadores: al décimo año de carrera (30-40 años) y próximo a la edad de jubilación (50 años).

Por otra parte, Cole (1979) analizó una muestra representativa de investigadores académicos en seis disciplinas, considerando los artículos publicados entre 1965 y 1969 y sus respectivas citas bibliográficas. Sus resultados indican que existe una relación curvilínea entre la productividad y la edad de los científicos, y que además existe un incremento de la productividad a los 30 años, mismo que empieza a declinar después de los 50 años. Finalmente, González-Brambila y Veloso (2007), empleando una muestra de los investigadores mexicanos pertenecientes al SNI en el periodo de 1991 a 2002, encontraron que existe una relación directa entre la edad y la productividad y que, a diferencia de los investigadores en otros países, los científicos mexicanos tienen un desempeño más prolongado, al reportar una edad máxima de productividad a los 53 años.

\section{Tamaño de la universidad}

Un factor importante en relación con la productividad de los investigadores es el tamaño de su institución de adscripción. Intuitivamente, se pensaría que una IES de mayor dimensión es sinónimo de mayores recursos, más capacidad de investigación, mayor capital humano, etc., sin embargo, debido a factores como la forma de gobierno de las universidades, podría no ser así. Behymer (1974) estudió la productividad en investigación en un periodo de cuatro años en colegios y en universidades, y encontró que las facultades en las universidades reportan mayor productividad en términos de artículos que los colegios.

Por su parte, Jordan et al. (1988) examinaron la relación entre productividad y la dimensión de la facultad en instituciones públicas y privadas de 
Estados Unidos, para lo cual emplearon como medida de productividad el promedio de artículos por facultad. Entre sus resultados destaca que las instituciones privadas son más fructíferas y que existe una relación positiva entre el tamaño de la institución y la productividad.

Finalmente, Crewe (1988) analizó la productividad en facultades de 52 universidades del Reino Unido de 1978 a 1984, y mostró que existe una fuerte variación de las tasas productividad entre facultades, lo cual atribuye a las diferencias en recursos y oportunidades de investigación entre dichas instancias.

\section{Datos}

Los datos empleados en este análisis provienen de un concurso (o match)realizado mediante la comparación de autores, instituciones, edad, genero, IES de adscripción y país de obtención del grado de doctor, realizada entre dos bases de datos. La primera fuente de información corresponde a datos procedentes del Science and Social Sciences Citation Index, desarrollados por el Institute of Scientific Information (ISI, 2012) (hoy Thomson Reuters Web of Knowledge), en la que se consideraron los artículos y las citas bibliográficas de todos los artículos científicos que cuenten al menos un autor con dirección de adscripción en México, publicados en el periodo 1991-2011, cabe mencionar que también se incluyó lo referente a la base de datos Arts and Humanities Citation Index.

La segunda fuente de información corresponde a una base de datos de 27,667 investigadores mexicanos de todas las áreas del conocimiento que han sido parte del sin, al menos durante un año, en el periodo 1991-2011. Para realizar el match fue necesario normalizar a los autores pertenecientes al SNI y buscar sus artículos en la base de datos de la WoS. La base de datos elaborada integra siete factores en relación con los investigadores:

a) Nombre.

b) Género.

c) Edad.

d) IES de adscripción.

e) Área del conocimiento de la especialidad.

f) País de obtención del doctorado.

g) Artículos y citas bibliográficas obtenidas de 1991 a 2011.

En esta base de datos se registraron 778 IES con al menos un artículo por institución, durante el periodo mencionado, de las cuales 390 son IES nacionales y 388 internacionales. Respecto de las nacionales, 148 son privadas (43 
universidades, 6 centros de investigación, 68 empresas y 31 asociaciones civiles) y 242 son de carácter público (117 universidades, 6 centros de investigación, 26 centros Conacyt, 81 entidades de gobierno y 12 institutos nacionales de salud).

Por otra parte, de las 388 instituciones internacionales, 329 son universidades, 10 centros de investigación, 30 empresas, 10 entidades gubernamentales y 9 son asociaciones civiles.

\section{CONSIDERACIONES METODOLÓGiCAS}

\section{Clasificación (ranking)}

Para elaborar la clasificación correspondiente a las cien IES con mayor número de artículos publicados y con más citas bibliográficas, en la base de datos únicamente se integraron las instituciones cuya misión es la educación superior. Adicionalmente, las empresas, entidades gubernamentales y asociaciones civiles también se eliminaron. Finalmente, la base de datos estudiada cuenta con 199 IES, de las cuales 149 son públicas y 47, privadas.

A fin de evitar la duplicidad en el conteo de los artículos por lugar de adscripción, sólo se consideró un artículo por IES, cuando éste cuente con más de dos autores de una misma institución. Por otra parte, la cantidad total de artículos por IES corresponde a la sumatoria de todos los artículos publicados de 1991 al 2011. Respecto de la clasificación de citas bibliográficas, el total corresponde a la sumatoria de las citas recibidas por cada artículo publicado por parte de la IES en el mismo periodo.

\section{TAMAÑO Y CLASIFICACIÓN DE LA IES}

Como describen Galaz (1998) y Grediaga (2006), no existe una clasificación de las IES mexicanas que se considere un patrón de referencia único para la elaboración de análisis relacionados con la educación superior, por lo que el uso de criterios inadecuados en su elaboración ha dificultado la comparación entre las IES. En este análisis se conformó una base de datos que permite determinar el tamaño de la IES, considerando cinco datos en relación con las instituciones:

1) Matrícula.

2) Número de profesores de tiempo completo (PTC).

3) Número de miembros del SNI.

4) Programas académicos. 
5) Número de programas de posgrado pertenecientes al Programa $\mathrm{Na}$ cional de Posgrado de Calidad PNPC.

Toda la información se analizó hasta el año 2011, debido a que, conforme a lo descrito en la página web Execum de la Coordinación de Planeación, Presupuesto y Evaluación de la UNAM, únicamente existe información de 2007 a 2016. Sin embargo, debido a que el periodo de análisis de nuestra investigación termina en 2011, dicho año se consideró como límite.

\section{Resultados}

En este trabajo se obtuvo información de 146,066 artículos publicados por investigadores mexicanos en el periodo 1991-2011, de los cuales el $83 \%$ fueron escritos por al menos un investigador que formó parte del SNI en dicho periodo (122,406 artículos). Sin embargo, únicamente el 53\% (81,530) de los trabajos recibieron al menos una cita bibliográfica.

En la gráfica 1 se muestra la producción anual promedio de los miembros del sin, acorde con el género de los investigadores. En dicha gráfica se observa un incremento importante en el promedio anual de artículos en ambos casos, aunque nuestros resultados indican que los hombres tienen mayor productividad que las mujeres: $75 \%$ y $25 \%$, respectivamente. Sin embargo, el promedio anual del periodo de estudio (21 años), indica que los investigadores reportan 0.56 artículos publicados anualmente (en promedio), mientras que las investigadoras registran 0.39 trabajos publicados, es decir, una diferencia 0.17 artículos por año.

Gráfica 1. Promedio anual de artículos por género

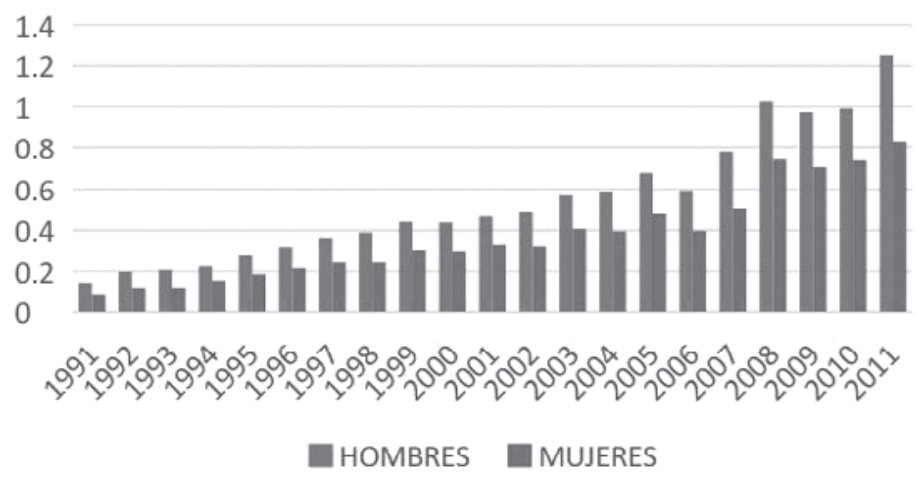


En cuanto a la influencia que tiene la edad de los investigadores en el nivel de productividad, nuestros resultados reflejan que los científicos mexicanos adscritos al SNI reportan un máximo en su productividad después de los cincuenta años, lo cual confirma lo descrito en trabajos previos como los de Bayer y Dutton (1977) y González-Brambila y Veloso (2007).

En la gráfica 2 se observa que los investigadores que han pertenecido al SNI alcanzan, en promedio, una productividad máxima a los 56 años de edad, aunque, considerando el género, las investigadoras alcanzan primero dicho máximo respecto de los investigadores (52 y 57 años, respectivamente).

Gráfica 2. Edad máxima de productividad de los miembros del SNI

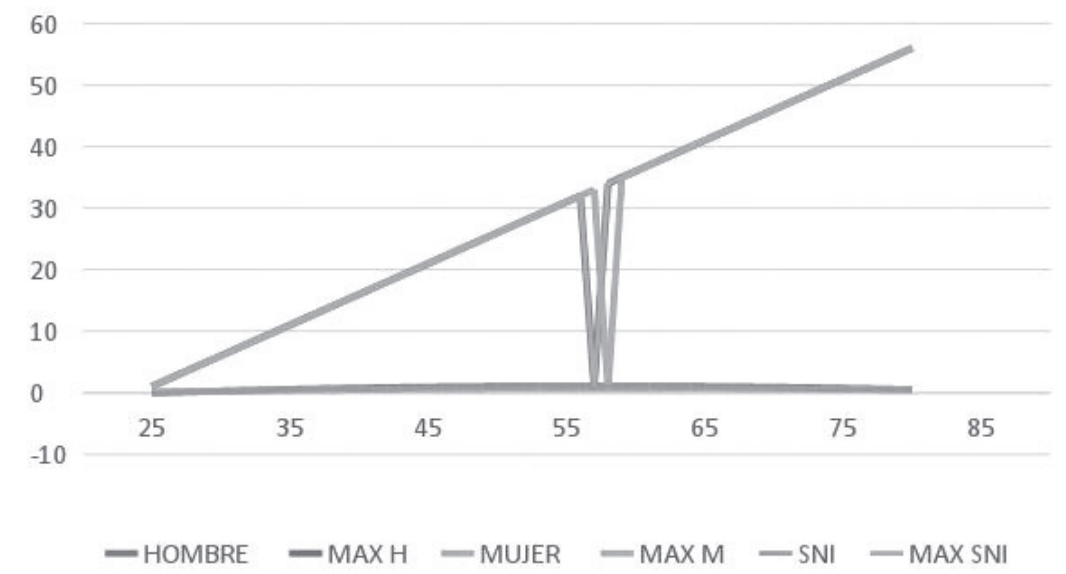

En la gráfica 3 se muestra el número de artículos publicados por los investigadores mexicanos, a lo largo de las dos décadas de estudio. Respecto de los trabajos que implican actividades de colaboración a nivel nacional, la cifra se incrementó de 1,046 artículos en 1991 a 6,645 en 2011; mientras que la colaboración con investigadores de otros países aumentó de 522 artículos en 1991 a 5,041 artículos científicos en 2011.

En cuanto a los trabajos publicados por un solo autor, únicamente se observó un pequeño incremento: 344 artículos publicados en 1991 y 898 artículos en 2011. En términos porcentuales, en 2011 la colaboración nacional representó el 52\% de los artículos publicados; la colaboración internacional el $40 \%$ y los artículos con un solo autor representaron únicamente el $7 \%$. Cabe señalar que el número de autores por artículo también aumentó considerablemente, ya que en 1991 se registró un promedio de 3.33 autores por 
artículo, mientras que en 2011 fue de 26 coautores. Sin embargo, conviene considerar que ese año se registraron 10 artículos con más de 2,000 autores (área de física), lo cual influyó en el promedio de coautoría.

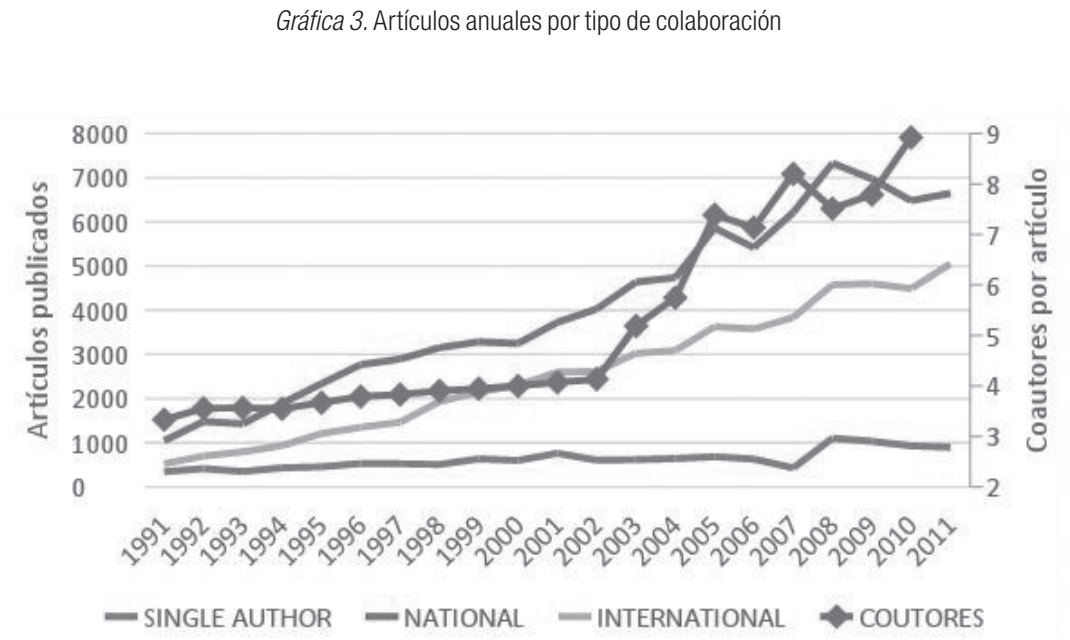

Nuestros resultados también reflejan que la producción promedio anual por investigador ha incrementado a lo largo del tiempo, observable esto mismo en la gráfica 4. La producción nacional en 1991 fue de 0.07 artículos por año y en 2011 se incrementó a 0.62 artículos anualmente. En cuanto a la producción internacional, fue de 0.02 artículos por año en 1991 y de 0.356 artículos en 2011.

Por último, los artículos de un solo autor se mantuvieron prácticamente sin cambios y con un promedio de 0.02 artículos por año. De los datos antes descritos se infiere que, hasta 2011, un investigador consiguió producir un artículo con coautoría nacional en aproximadamente un año y medio, un artículo con coautoría internacional en casi tres años y un artículo sin coautoría en más de diez años. 


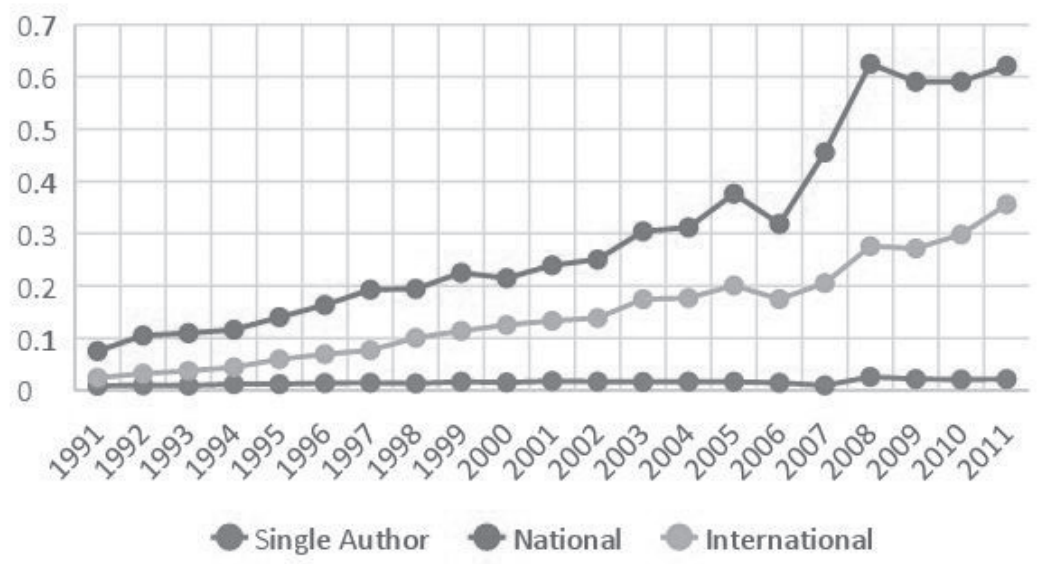

Con el propósito de conocer si una IES se encuentra más orientada a la investigación o a la docencia, en los Cuadros 2 y 3 de este trabajo se muestra el escalafón de las cien IES en México con mayor número de artículos publicados y de citas bibliográficas, respectivamente. En ambos cuadros, el periodo de análisis es de 1991-2011. Cabe considerar que, en los dos casos, a medida que una IES se ubica en los primeros lugares, indica que está más orientada a la investigación y, conforme la posición desciende, significa que la IES está más orientada a la docencia.

Los resultados muestran que las diez universidades más productivas en términos del número de artículos son, a su vez, las que cuentan con el mayor número de citas bibliográficas. Conviene señalar aquí que cuatro son IES federales, seis estatales y que todas tienen un gobierno autónomo, a excepción del Instituto Politécnico Nacional (IPN).

El Cuadro 2 muestra la clasificación nacional de las cien IES con mayor número de artículos publicados en el periodo 1991-2011. Se observa que la UNAM se ubica en el primer lugar del cuadro, con 45,397 trabajos (37\% del total); en segundo lugar está el Cinvestav, del IPN, con 14,168 artículos (11.5 $\%$ ); en tercer lugar, se ubica el Instituto Politécnico Nacional, con 9,546 artículos (7.7\%); en cuarto lugar se ubica la Universidad Autónoma Metropolitana (UAM), con 8,271 artículos (6.75\%); en quinto lugar, la Universidad de Guadalajara (UDG) con 3,975 artículos (3.24\%), en sexto lugar se posiciona la Universidad Autónoma de Nuevo León (UANL), con 3,921 trabajos 
de Puebla (BUAP), con 3728 artículos (3.1\%); en octavo lugar, la Universidad de Guanajuato (UGTO), con 2,953 artículos (2.4\%); en noveno la Universidad Autónoma de San Luis Potosí (UASLP), con 2,865 trabajos (2.3\%) y en décimo lugar el Instituto Nacional de Astrofísica, Óptica y Electrónica (INAOE), con 2,751 artículos científicos (2.2\%). El lugar número 100 lo ocupa el Centro Nacional de Investigación y Desarrollo Tecnológico (Cenidet), con 23 artículos, lo que equivale al $0.018 \%$ de la producción nacional. Adicionalmente, nuestros resultados indican que en México sólo dos instituciones reportaron una productividad mayor a diez mil artículos, publicados entre 1991 y 2011.

Cuadro 2. Top 100 de las IES por artículos durante 1991-2011

\begin{tabular}{|l|l|l|l|l|l|l|l|l|}
\hline Rank & \multicolumn{1}{|c|}{ IES } & \multicolumn{1}{|c|}{ Pubs } & \multicolumn{1}{|c|}{ Rank } & \multicolumn{1}{|c|}{ IES } & \multicolumn{1}{|c|}{ Pubs } & \multicolumn{1}{|c|}{ Rank } & \multicolumn{1}{|c|}{ IES } & Pubs \\
\hline 1 & UNAM & 45,397 & 36 & Ibero & 635 & 71 & UAN & 98 \\
\hline 2 & Cinvestav & 14,168 & 37 & Cimat & 621 & 72 & UPP & 96 \\
\hline 3 & IPN & 9,546 & 38 & CIQA & 544 & 73 & UNICAH & 82 \\
\hline 4 & UAM & 8,271 & 39 & Colmex & 538 & 74 & ITESO & 80 \\
\hline 5 & UDG & 3,975 & 40 & UAT & 511 & 75 & UABD & 78 \\
\hline 6 & UANL & 3,921 & 41 & UAAAN & 487 & 76 & Cidesi & 65 \\
\hline 7 & BUAP & 3,798 & 42 & UACH & 473 & 77 & USAUE & 72 \\
\hline 8 & UGTO & 2,953 & 43 & ITAM & 464 & 78 & Udefa & 64 \\
\hline 9 & UASIP & 2,865 & 44 & UAA & 402 & 79 & UAM & 63 \\
\hline 10 & INAOE & 2,751 & 45 & UABCS & 395 & 80 & UPN & 60 \\
\hline 11 & UAEM & 2,718 & 46 & UED & 365 & 81 & CiateC & 58 \\
\hline 12 & UMICH & 2,352 & 47 & UADEC & 359 & 82 & UPEM OR & 55 \\
\hline 13 & Cicese & 2,343 & 48 & UPAEP & 351 & 83 & Comimsa & 54 \\
\hline 14 & USON & 1,887 & 49 & UACM & 340 & 84 & UDO & 44 \\
\hline 15 & COIpOS & 1,815 & 50 & UACAM & 332 & 85 & ENAH & 44 \\
\hline 16 & Cibnor & 1,623 & 51 & UAAT & 324 & 86 & UNISTM0 & 44 \\
\hline 17 & ITESM & 1,611 & 52 & UATX & 318 & 87 & Cesues & 41 \\
\hline 18 & UV & 1,555 & 53 & UACJ & 300 & 88 & UPSIP & 41 \\
\hline 19 & INECOL & 1,528 & 54 & CIDE & 249 & 89 & UPV & 37 \\
\hline 20 & UABC & 1,486 & 55 & Cideteq & 248 & 90 & CoImich & 35 \\
\hline 21 & CIO & 1,445 & 56 & CIATEJ & 236 & 91 & UDEM & 35 \\
\hline 22 & UAEMEX & 1,287 & 57 & Colef & 212 & 92 & Marista & 29 \\
\hline
\end{tabular}




\begin{tabular}{|l|l|l|l|l|l|l|l|l|}
\hline Rank & \multicolumn{1}{|c|}{ IES } & \multicolumn{1}{|c|}{ Pubs } & \multicolumn{1}{c|}{ Rank } & \multicolumn{1}{|c|}{ IES } & \multicolumn{1}{c|}{ Pubs } & \multicolumn{1}{c|}{ Rank } & \multicolumn{1}{c|}{ IES } & Pubs \\
\hline 23 & ECOSUr & 1,208 & 58 & UAN & 185 & 93 & UCEM & 28 \\
\hline 24 & UAEH & 1,203 & 59 & UAGPO & 185 & 94 & UPPUE & 28 \\
\hline 25 & CIAD & 1,136 & 60 & UTM & 164 & 95 & UPTAX & 27 \\
\hline 26 & UAQ & 1,127 & 61 & ITSON & 161 & 96 & FlaCSO & 26 \\
\hline 27 & UADY & 1,077 & 62 & UP & 149 & 97 & TESE & 26 \\
\hline 28 & UCOL & 970 & 63 & UM AR & 142 & 98 & UTP & 24 \\
\hline 29 & IPICYT & 919 & 64 & UNACAR & 137 & 99 & IM OPA & 24 \\
\hline 30 & UDLAP & 884 & 65 & UNPA & 133 & 100 & Cenidet & 23 \\
\hline 31 & UAS & 851 & 66 & UNACH & 123 & & & \\
\hline 32 & CICY & 843 & 67 & UAG & 114 & & & \\
\hline 33 & UAZ & 840 & 68 & Ciateq & 111 & & & \\
\hline 34 & UACH & 788 & 69 & UVM & 109 & & & \\
\hline 35 & Cimav & 761 & 70 & CIESAS & 99 & & & \\
\hline
\end{tabular}

Fuente: elaboración propia.

En otro orden, las IES que se encuentran entre el tercer y décimo lugar de la clasificación publicaron entre 3,000 y 9,000 artículos; del lugar 14 al 27 reportan un rango de 1,000 a 2,000 mil artículos y, finalmente, el resto de las IES (73) obtuvieron una productividad menor a 1,000 artículos publicados.

En el Cuadro 3 se muestran las cien IES con mayor número de citas bibliográficas recibidas en sus artículos publicados. En dicho cuadro se observa que lasdiez IES que ocupan los primeros lugares corresponden a las mismas instituciones que se ubican entre los primeros lugares de la clasificación de artículos publicados: la UNAM ocupa el primer lugar con 459,426 citas bibliográficas; el Cinvestav se ubica en el segundo lugar (127,879), posteriormente la UAM en tercer lugar $(63,951)$; en cuarto lugar el IPN $(57,316)$; en quinto lugar la BUAP $(28,520)$; en sexto lugar el INAOE $(25,417)$; en séptimo la UGTO $(24,456)$; en octavo la UANL $(24,214)$; la uadg en noveno lugar $(22,249)$ y en décimo se ubica la UASLP $(21,186)$. Finalmente, el Cenidet (lugar 100 en artículos) ocupa el lugar 79 del conteo con 328 citas bibliográficas. 
EL SISTEMA NACIONAL DE INVESTIGADORES EN MÉXICO...

Cuadro 3. Top cien de las IES por citas bibliográficas (1991-2011)

\begin{tabular}{|c|c|c|c|c|c|c|c|c|}
\hline Rank & IES & Citas biblio. & Rank & IES & $\begin{array}{l}\text { Citas } \\
\text { biblio. }\end{array}$ & Rank & IES & $\begin{array}{l}\text { Citas } \\
\text { biblio. }\end{array}$ \\
\hline 1 & UNAM & 459,426 & 36 & Ibero & 3,865 & 71 & UPEM OR & 434 \\
\hline 2 & Cinvestav & 127,879 & 37 & $\mathrm{UACH}$ & 3,350 & 72 & UPTAXX & 395 \\
\hline 3 & UAM & 63,951 & 38 & ITAM & 2,967 & 73 & uiim & 367 \\
\hline 4 & IPN & 60,924 & 39 & UABCS & 2,892 & 74 & UPP & 365 \\
\hline 5 & BUAP & 28,520 & 40 & CIQA & 2,844 & 75 & CIESAS & 350 \\
\hline 6 & INAOE & 25,417 & 41 & UADEC & 2,746 & 76 & UTM & 346 \\
\hline 7 & UGTO & 24,456 & 42 & UAT & 2,654 & 77 & ciateq & 343 \\
\hline 8 & UANL & 24,214 & 43 & $\mathrm{UACH}$ & 2,524 & 78 & USAUE & 329 \\
\hline 9 & UDG & 22,249 & 44 & UPAEP & 2,308 & 79 & Cenidet & 328 \\
\hline 10 & UASIP & 21,186 & 45 & UATX & 2,124 & 80 & UDO & 293 \\
\hline 11 & UAEM & 20,182 & 46 & Colef & 2,057 & 81 & ITESO & 272 \\
\hline 12 & Cicese & 19,359 & 47 & UED & 1,968 & 82 & Cidesi & 251 \\
\hline 13 & UMICH & 17,139 & 48 & UACM & 1,882 & 83 & Marista & 250 \\
\hline 14 & Cibnor & 13,449 & 49 & UAA & 1,881 & 84 & UABD & 227 \\
\hline 15 & INECOL & 12,040 & 50 & UAAAN & 1,761 & 85 & ciatec & 219 \\
\hline 16 & USON & 11,373 & 51 & UACAM & 1,755 & 86 & UPN & 209 \\
\hline 17 & $\mathrm{ClO}$ & 10,233 & 52 & UAT & 1,552 & 87 & UNICAH & 193 \\
\hline 18 & UV & 9,246 & 53 & Cideteq & 1,441 & 88 & ENAH & 191 \\
\hline 19 & Ecosur & 9,117 & 54 & UAN & 1,381 & 89 & UDEM & 181 \\
\hline 20 & IPICYT & 9,097 & 55 & UVM & 1,280 & 90 & UCEM & 179 \\
\hline 21 & CIAD & 8,835 & 56 & UACJ & 1,150 & 91 & TESE & 152 \\
\hline 22 & UAEH & 8,277 & 57 & CIDE & 1,042 & 92 & utsjr & 126 \\
\hline 23 & ITESM & 8,108 & 58 & CIATEJ & 962 & 93 & Cesues & 125 \\
\hline 24 & UABC & 8,101 & 59 & UP & 905 & 94 & upa & 120 \\
\hline 25 & UAS & 7,942 & 60 & UAG & 834 & 95 & UPSIP & 110 \\
\hline 26 & UAQ & 7,713 & 61 & ITSON & 829 & 96 & utl & 109 \\
\hline 27 & COLPOS & 7,003 & 62 & Colmex & 642 & 97 & csaegro & 97 \\
\hline 28 & UADY & 6,291 & 63 & udefa & 636 & 98 & Flacso & 97 \\
\hline 29 & CICY & 6,015 & 64 & UNACH & 613 & 99 & UPPUE & 81 \\
\hline 30 & UCOL & 5,877 & 65 & UAN & 574 & 100 & unsij & 81 \\
\hline 31 & UDLAP & 5,691 & 66 & UM AR & 551 & & & \\
\hline 32 & UAZ & 5,409 & 67 & UAGPD & 548 & & & \\
\hline
\end{tabular}




\begin{tabular}{|l|l|l|l|l|l|l|l|l|}
\hline Rank & IES & Citas biblio. & Rank & IES & $\begin{array}{r}\text { Citas } \\
\text { biblio. }\end{array}$ & Rank & IES & $\begin{array}{c}\text { Citas } \\
\text { biblio. }\end{array}$ \\
\hline 33 & Cimat & 5,220 & 68 & UAM & 491 & & & \\
\hline 34 & UAEMEX & 5,127 & 69 & UNPA & 458 & & & \\
\hline 35 & Cimav & 4,077 & 70 & UNACAR & 443 & & & \\
\hline
\end{tabular}

Fuente: elaboración propia.

CITAS BIBLIO = número de citas bibliográficas recibidas.

En el Cuadro 4 se muestra la diferencia del promedio anual del número de ingresos y del número de bajas de los investigadores al SNI ( $\Delta$ sni) de cada IES, la cual está ordenada de forma ascendente, conforme a dicho gradiente. El valor de la columna Altas corresponde al promedio del número total de investigadores que ingresaron al SNI por año en el periodo de 1991 a 2011; el valor de la columna Bajas se obtuvo al promediar el número total de investigadores que causaron baja en el sistema por año en el periodo mencionado; la columna SNI corresponde al promedio de investigadores vigentes en el sistema por año de 1991 a 2011, mientras que la columna correspondiente al Impacto indica el porcentaje que representan las bajas en relación con el número de investigadores (SNI). Finalmente, la columna Rank corresponde a la posición que ocupa la institución en el conteo de las cien IES más productivas.

Cuadro 4. Promedio de altas y bajas del SNI de 1991 a 2011 por institución

\begin{tabular}{|l|l|l|l|l|l|l|}
\hline \multicolumn{1}{|c|}{$\Delta$ SNI } & \multicolumn{1}{c|}{ IES } & \multicolumn{1}{c|}{ Altas } & \multicolumn{1}{c|}{ Bajas } & \multicolumn{1}{c|}{ SNI } & \multicolumn{1}{c|}{ Impacto } & \multicolumn{1}{c|}{ Rank $^{*}$} \\
\hline-0.13 & TESE & 1.00 & 1.13 & 4.25 & 26.47 & 97 \\
\hline 0.13 & CIATEC & 0.75 & 0.63 & 5.63 & 11.11 & 81 \\
\hline 0.25 & UPTLAX & 0.50 & 0.25 & 2.00 & 12.50 & 95 \\
\hline 0.38 & UPPUE & 1.13 & 0.75 & 2.88 & 26.09 & 94 \\
\hline 0.37 & Cenidet & 2.50 & 2.12 & 14.25 & 14.91 & 100 \\
\hline 0.38 & Marista & 0.38 & 0.00 & 1.75 & 0.00 & 92 \\
\hline 0.38 & UAM & 0.38 & 0.00 & 1.63 & 0.00 & 79 \\
\hline 0.63 & UCEM & 0.75 & 0.13 & 1.38 & 9.09 & 93 \\
\hline 0.63 & UVM & 1.13 & 0.50 & 3.13 & 16.00 & 69 \\
\hline 0.63 & UPV & 0.75 & 0.13 & 1.88 & 6.67 & 89 \\
\hline 0.63 & UPSLP & 1.25 & 0.63 & 3.13 & 20.00 & 88 \\
\hline 0.63 & Cesues & 0.88 & 0.25 & 3.63 & 6.90 & 87 \\
\hline 0.75 & UDEFA & 1.00 & 0.25 & 3.38 & 7.41 & 78 \\
\hline 0.88 & Cidesi & 1.63 & 0.75 & 5.88 & 12.77 & 76 \\
\hline
\end{tabular}


EL SISTEMA NACIONAL DE INVESTIGADORES EN MÉXICO...

\begin{tabular}{|c|c|c|c|c|c|c|}
\hline$\Delta \mathrm{SNI}$ & IES & Altas & Bajas & SNI & Impacto & Rank $^{*}$ \\
\hline 0.88 & UPEMOR & 1.00 & 0.13 & 1.25 & 10.00 & 82 \\
\hline 0.88 & ENAH & 2.63 & 1.75 & 30.88 & 5.67 & 85 \\
\hline 0.88 & UNISTMO & 1.63 & 0.75 & 5.25 & 14.29 & 86 \\
\hline 0.88 & Comimsa & 1.25 & 0.38 & 3.63 & 10.34 & 83 \\
\hline 1.00 & Cideteq & 1.25 & 0.25 & 14.13 & 1.77 & 55 \\
\hline 1.13 & UPP & 3.88 & 2.75 & 13.50 & 20.37 & 72 \\
\hline 1.25 & USALLE & 1.88 & 0.63 & 5.00 & 12.50 & 77 \\
\hline 1.25 & UDEM & 1.38 & 0.13 & 7.13 & 1.75 & 91 \\
\hline 1.25 & UAG & 1.25 & 0.00 & 5.75 & 0.00 & 67 \\
\hline 1.38 & Ciateq & 2.25 & 0.88 & 12.63 & 6.93 & 68 \\
\hline 1.38 & Flacso & 2.38 & 1.00 & 20.25 & 4.94 & 96 \\
\hline 1.38 & UPAEP & 2.50 & 1.13 & 12.88 & 8.74 & 48 \\
\hline 1.63 & ITESO & 2.38 & 0.75 & 18.13 & 4.14 & 74 \\
\hline 1.75 & UABJO & 3.13 & 1.38 & 16.00 & 8.59 & 75 \\
\hline 1.75 & UDO & 2.13 & 0.38 & 7.38 & 5.08 & 84 \\
\hline 2.00 & UABCS & 4.13 & 2.13 & 26.63 & 7.98 & 45 \\
\hline 2.13 & UNICAH & 2.50 & 0.38 & 12.38 & 3.03 & 73 \\
\hline 2.50 & UDLAP & 7.75 & 5.25 & 66.63 & 7.88 & 30 \\
\hline 2.50 & UMAR & 4.88 & 2.38 & 20.00 & 11.88 & 63 \\
\hline 2.50 & Colmich & 4.00 & 1.50 & 42.00 & 3.57 & 90 \\
\hline 2.50 & CIESAS & 4.50 & 2.00 & 106.25 & 1.88 & 70 \\
\hline 2.63 & ITSON & 3.13 & 0.50 & 13.00 & 3.85 & 61 \\
\hline 2.63 & $\mathrm{ClO}$ & 3.5 & 0.88 & 58.88 & 1.49 & 21 \\
\hline 2.75 & CIQA & 3.88 & 1.13 & 37.63 & 2.99 & 38 \\
\hline 2.75 & UAAAN & 4.13 & 1.38 & 41.38 & 3.32 & 41 \\
\hline 2.75 & ITAM & 8.13 & 5.38 & 69.38 & 7.75 & 43 \\
\hline 2.88 & UJED & 5.25 & 2.38 & 25.00 & 9.50 & 46 \\
\hline 3.00 & СIMAT & 4.13 & 1.13 & 53.00 & 2.12 & 37 \\
\hline 3.00 & UACAM & 4.75 & 1.75 & 26.75 & 6.54 & 50 \\
\hline 3.13 & UNACAR & 4.25 & 1.13 & 14.25 & 7.89 & 64 \\
\hline 3.50 & Colmex & 6.25 & 2.75 & 155.25 & 1.77 & 39 \\
\hline 3.50 & CIBNOR & 8.00 & 4.50 & 95.88 & 4.69 & 16 \\
\hline 3.50 & UAN & 4.75 & 1.25 & 14.00 & 8.93 & 58 \\
\hline 3.63 & UNPA & 4.63 & 1.00 & 12.25 & 8.16 & 65 \\
\hline 4.00 & Cicese & 9.50 & 5.50 & 141.75 & 3.88 & 13 \\
\hline
\end{tabular}




\begin{tabular}{|c|c|c|c|c|c|c|}
\hline$\Delta \mathrm{SNI}$ & IES & Altas & Bajas & SNI & Impacto & Rank* $^{*}$ \\
\hline 4.13 & UTM & 5.13 & 1.00 & 15.5 & 6.45 & 60 \\
\hline 4.13 & Colef & 6.00 & 1.88 & 65.38 & 2.87 & 57 \\
\hline 4.13 & UP & 5.63 & 1.50 & 29.13 & 5.15 & 62 \\
\hline 4.25 & UAA & 7.25 & 3.00 & 45.38 & 6.61 & 44 \\
\hline 4.25 & UADEC & 7.13 & 2.88 & 38.63 & 7.44 & 47 \\
\hline 4.25 & CIATEJ & 6.13 & 1.88 & 25.75 & 7.28 & 56 \\
\hline 4.50 & CIDE & 6.25 & 1.75 & 58.75 & 2.98 & 54 \\
\hline 4.75 & INECOL & 7.38 & 2.63 & 75.13 & 3.49 & 19 \\
\hline 5.00 & IPICYT & 6.25 & 1.25 & 50.13 & 2.49 & 29 \\
\hline 5.13 & UAGRO & 8.38 & 3.25 & 27.25 & 11.93 & 59 \\
\hline 5.25 & INAOE & 7.88 & 2.63 & 103.88 & 2.53 & 10 \\
\hline 5.50 & Cimav & 6.25 & 0.75 & 45.13 & 1.66 & 35 \\
\hline 5.50 & Ibero & 8.63 & 3.13 & 79.25 & 3.94 & 36 \\
\hline 5.75 & UJAT & 10.13 & 4.38 & 42.00 & 10.42 & 51 \\
\hline 5.75 & UATX & 8.50 & 2.75 & 39.50 & 6.96 & 52 \\
\hline 5.75 & UNACH & 7.25 & 1.50 & 28.75 & 5.22 & 66 \\
\hline 6.13 & $\mathrm{UACH}$ & 8.88 & 2.75 & 35.63 & 7.72 & 34 \\
\hline 6.13 & UACH & 8.88 & 2.75 & 35.63 & 7.72 & 43 \\
\hline 6.38 & CICY & 8.88 & 2.50 & 64.50 & 3.88 & 32 \\
\hline 6.38 & CIAD & 11.13 & 4.75 & 84.88 & 5.60 & 25 \\
\hline 6.63 & Ecosur & 9.88 & 3.25 & 86.75 & 3.75 & 42 \\
\hline 7.00 & UAT & 11.38 & 4.38 & 52.88 & 8.27 & 40 \\
\hline 7.13 & UPN & 9.75 & 2.63 & 49.38 & 5.32 & 80 \\
\hline 8.13 & UACM & 10.38 & 2.25 & 38.25 & 5.88 & 50 \\
\hline 9.75 & Colpos & 19.13 & 9.38 & 214.38 & 4.37 & 15 \\
\hline 10.25 & UAQ & 15.38 & 5.13 & 94.50 & 5.42 & 26 \\
\hline 10.25 & UAS & 16.75 & 6.50 & 93.88 & 6.92 & 31 \\
\hline 10.50 & UACJ & 12.88 & 2.38 & 47.38 & 5.01 & 53 \\
\hline 10.63 & UADY & 17.50 & 6.88 & 116.88 & 5.88 & 27 \\
\hline 10.63 & UCOL & 17.00 & 6.38 & 94.38 & 6.75 & 28 \\
\hline 10.75 & UAEM & 21.00 & 10.25 & 192.38 & 5.33 & 11 \\
\hline 11.13 & UAZ & 16.25 & 5.13 & 90.88 & 5.64 & 33 \\
\hline 15.13 & USON & 22.88 & 7.75 & 150.00 & 5.17 & 14 \\
\hline 17.13 & UAEH & 26.75 & 9.63 & 142.63 & 6.75 & 24 \\
\hline 19.88 & BUAP & 35.38 & 15.5 & 307.13 & 5.05 & 7 \\
\hline
\end{tabular}




\begin{tabular}{|l|l|l|l|l|l|l|}
\hline \multicolumn{1}{|c|}{$\Delta$ SNI } & \multicolumn{1}{c|}{ IES } & \multicolumn{1}{c|}{ Altas } & \multicolumn{1}{c|}{ Bajas } & \multicolumn{1}{c|}{ SNI } & \multicolumn{1}{c|}{ Impacto } & \multicolumn{1}{c|}{ Rank $^{*}$} \\
\hline 20.13 & UV & 29.00 & 8.88 & 187.25 & 4.74 & 18 \\
\hline 20.50 & UABC & 28.13 & 7.63 & 149.63 & 5.10 & 20 \\
\hline 21.38 & UMICH & 31.63 & 10.25 & 230.00 & 4.46 & 12 \\
\hline 21.50 & UASLP & 29.75 & 8.25 & 184.25 & 4.48 & 9 \\
\hline 22.50 & UGTO & 29.25 & 6.75 & 188.38 & 3.58 & 8 \\
\hline 24.50 & ITESM & 47.13 & 22.63 & 230.38 & 9.82 & 17 \\
\hline 25.63 & UAEMEX & 33.13 & 7.50 & 180.63 & 4.15 & 22 \\
\hline 29.00 & UINVESTAV & 43.63 & 14.63 & 583.88 & 2.50 & 2 \\
\hline 34.25 & UANL & 51.13 & 16.88 & 297.13 & 5.68 & 6 \\
\hline 45.00 & UAM & 72.88 & 27.88 & 754.25 & 3.70 & 4 \\
\hline 48.75 & UDG & 71.88 & 23.13 & 481.25 & 4.81 & 5 \\
\hline 53.25 & IPN & 79.13 & 25.88 & 562.38 & 4.60 & 3 \\
\hline 139.25 & UNAM & 240.13 & 100.88 & 3147.63 & 3.20 & 1 \\
\hline
\end{tabular}

* Lugar que ocupa en el conteo de las cien IES con mayor número de artículos de 1991 a 2011.

Fuente: elaboración propia.

Se observa que las instituciones con $\Delta$ sni bajo son las que se encuentran en los últimos lugares de la clasificación de artículos (Cuadro 2) y que, en la mayoría de los casos, el impacto es alto. Por ejemplo, la IES con menor $\Delta$ sni (-0.13) es el TESE, institución que en los 21 años del periodo de estudio en promedio reporta un alta y 1.13 bajas por año al sistema, el número de bajas representan el $26 \%$ de total de sus investigadores y ocupa el lugar 97 en el conteo de artículos.

En otro orden, la UNAM (primer lugar de artículos) reporta el $\Delta$ sni más alto de todas las instituciones (139.25), tiene un promedio de 240 altas por año y 100 bajas del SNI y sus bajas representan tan sólo el $3.25 \%$ de su total de investigadores. Además, en este cuadro se observa que 8 de las 10 IES más productivas señaladas en el Cuadro 2, se ubican en los últimos lugares de acuerdo con el $\Delta$ sni obtenido (UNAM, IPN, UDG, UAM, UANL, Cinvestav, UGTO y UASLP).

En el Cuadro 5 se muestra el tamaño de las IES, con base en el número de programas de posgrado que tienen adscritos al pnpc. Se observa que la UNAM es la IES que cuenta con el mayor número de programas registrados en dicho programa (135) y que a nivel general cuenta con la mayor matrícula (204,581 alumnos), con el más elevado número de investigadores con distinción en el SNI $(4,918)$ y con el más alto índice de profesores de tiempo completo (3,583); lo que muestra que la UNAM es una IES con una fuerte tendencia a realizar investigación científica, pero sin perder el objetivo de la docencia. 


\begin{tabular}{|c|c|c|c|c|c|c|}
\hline \multicolumn{7}{|c|}{ Cuadro 5. Tamaño de las ies en 2011} \\
\hline$P N P C$ & IES & PTC & $S N I$ & Matrícula & $\begin{array}{l}\text { Prog } \\
\text { Acad }\end{array}$ & Rank* $^{*}$ \\
\hline 135 & UNAM & 4918 & 3,583 & 204,581 & 333 & 1 \\
\hline 81 & UDG & 3087 & 662 & 92,451 & 389 & 5 \\
\hline 73 & IPN & 4901 & 779 & 100,452 & 224 & 3 \\
\hline 72 & UANL & 2647 & 443 & 79,246 & 276 & 6 \\
\hline 63 & UAM & 3535 & 906 & 51,935 & 142 & 4 \\
\hline 56 & Cinvestav & 588 & 727 & 2,312 & 58 & 2 \\
\hline 49 & ITESM & 1506 & 264 & 72,151 & 1125 & 17 \\
\hline 42 & UASLP & 992 & 262 & 24,121 & 152 & 9 \\
\hline 42 & UV & 1640 & 299 & 58,944 & 245 & 18 \\
\hline 40 & UAEMEX & 1034 & 288 & 41,362 & 282 & 22 \\
\hline 36 & UMICH & 1277 & 311 & 37,411 & 103 & 12 \\
\hline 35 & BUAP & 1803 & 384 & 54,434 & 189 & 7 \\
\hline 33 & UABC & 1166 & 216 & 47,878 & 173 & 20 \\
\hline 27 & UGTO & 652 & 265 & 17,812 & 153 & 8 \\
\hline 25 & UAEM & 361 & 233 & 13,251 & 126 & 11 \\
\hline 24 & UACJ & 640 & 96 & 22,277 & 99 & 53 \\
\hline 22 & USON & 655 & 226 & 25,895 & 164 & 14 \\
\hline 21 & UACH & 597 & 114 & 4,612 & 46 & 34 \\
\hline 20 & UADY & 482 & 167 & 13,841 & 107 & 27 \\
\hline 20 & UAS & 1114 & 139 & 50,607 & 173 & 31 \\
\hline 19 & Cicese & 179 & 150 & 428 & 18 & 13 \\
\hline 19 & Colpos & 335 & 239 & 948 & 32 & 15 \\
\hline 18 & UAEH & 742 & 170 & 20,067 & 108 & 24 \\
\hline 18 & UAQ & 471 & 152 & 17,813 & 161 & 26 \\
\hline 18 & UADEC & 718 & 59 & 23,650 & 127 & 47 \\
\hline 15 & UAA & 392 & 68 & 12,718 & 85 & 44 \\
\hline 14 & UAT & 871 & 69 & 39,311 & 237 & 40 \\
\hline 13 & IPICYT & 0 & 63 & 0 & 0 & 29 \\
\hline 13 & UACH & 675 & 50 & 25,768 & 166 & 42 \\
\hline 12 & Colmex & 186 & 172 & 368 & 166 & 39 \\
\hline 11 & UCOL & 490 & 138 & 12,291 & 18 & 28 \\
\hline 11 & Ibero & 478 & 104 & 19,145 & 114 & 36 \\
\hline 11 & UAAAN & 497 & 56 & 5,193 & 227 & 41 \\
\hline
\end{tabular}


EL SISTEMA NACIONAL DE INVESTIGADORES EN MÉXICO...

\begin{tabular}{|c|c|c|c|c|c|c|}
\hline PNPC & IES & PTC & SNI & Matrícula & $\begin{array}{l}\text { Prog } \\
\text { Acad }\end{array}$ & Rank $^{*}$ \\
\hline 10 & CIESAS & 33 & 112 & 147 & 33 & 70 \\
\hline 9 & Colmich & 62 & 49 & 145 & 6 & 90 \\
\hline 8 & INAOE & 107 & 112 & 409 & 7 & 10 \\
\hline 7 & CICY & 73 & 89 & 242 & 8 & 32 \\
\hline 6 & UAZ & 1233 & 133 & 18,906 & 5 & 33 \\
\hline 5 & UABCS & 132 & 32 & 4,756 & 86 & 45 \\
\hline 5 & UATX & 654 & 62 & 11,144 & 37 & 52 \\
\hline 5 & Colef & 101 & 84 & 127 & 63 & 57 \\
\hline 5 & UNACH & 729 & 55 & 20,531 & 100 & 66 \\
\hline 4 & Cimav & 48 & 56 & 81 & 5 & 35 \\
\hline 4 & Cimat & 69 & 61 & 148 & 9 & 37 \\
\hline 4 & CIQA & 49 & 51 & 48 & 4 & 38 \\
\hline 4 & UAGRO & 787 & 37 & 25,214 & 81 & 59 \\
\hline 4 & Cidesi & 0 & 6 & 59 & 6 & 76 \\
\hline 4 & Flacso & 48 & 23 & 611 & 9 & 96 \\
\hline 3 & $\mathrm{ClO}$ & 57 & 62 & 64 & 3 & 21 \\
\hline 3 & Ecosur & 118 & 104 & 145 & 10 & 23 \\
\hline 3 & CIAD & 138 & 108 & 198 & 3 & 25 \\
\hline 3 & UJED & 370 & 49 & 13,906 & 88 & 46 \\
\hline 3 & UJAT & 931 & 55 & 27,626 & 92 & 51 \\
\hline 3 & CIDE & 110 & 67 & 378 & 6 & 54 \\
\hline 3 & USALLE & 329 & 11 & 27,043 & 380 & 77 \\
\hline 2 & CIBNOR & 91 & 111 & 146 & 2 & 16 \\
\hline 2 & UACAM & 215 & 42 & 5891 & 83 & 50 \\
\hline 2 & Cideteq & 25 & 19 & 64 & 4 & 55 \\
\hline 2 & UAN & 683 & 35 & 13,500 & 54 & 58 \\
\hline 2 & ITSON & 330 & 21 & 15,452 & 79 & 61 \\
\hline 2 & UAN & 683 & 35 & 13,500 & 54 & 71 \\
\hline 1 & ITAM & 198 & 69 & 5,365 & 30 & 43 \\
\hline 1 & UACM & 791 & 79 & 12,425 & 55 & 50 \\
\hline 1 & CIATEJ & 24 & 46 & 67 & 2 & 56 \\
\hline 1 & UNACAR & 218 & 24 & 4,511 & 74 & 64 \\
\hline 1 & UPP & 72 & 13 & 2,777 & 22 & 72 \\
\hline 1 & UPN & 1642 & 71 & 71,901 & 418 & 80 \\
\hline
\end{tabular}




\begin{tabular}{|c|c|c|c|c|c|c|}
\hline PNPC & IES & PTC & SNI & Matrícula & $\begin{array}{l}\text { Prog } \\
\text { Acad }\end{array}$ & Rank* $^{*}$ \\
\hline 1 & CIATEC & 0 & 4 & 0 & 0 & 81 \\
\hline 1 & Comimsa & 16 & 8 & 50 & 4 & 83 \\
\hline 1 & UPV & 17 & 5 & 681 & 4 & 89 \\
\hline 1 & UPPUE & 31 & 4 & 1,039 & 9 & 94 \\
\hline 1 & TESE & 142 & 9 & 5,500 & 13 & 97 \\
\hline 0 & INECOL & 51 & 88 & 134 & 2 & 19 \\
\hline 0 & UAG & 301 & 8 & 12,967 & 182 & 67 \\
\hline 0 & Ciateq & 0 & 19 & 0 & 0 & 68 \\
\hline 0 & UVM & 418 & 6 & 63,940 & 813 & 69 \\
\hline 0 & UABJO & 261 & 20 & 15,667 & 48 & 75 \\
\hline 0 & UDEFA & 1028 & 3 & 707 & 58 & 78 \\
\hline 0 & UPEMOR & 19 & 0 & 1,619 & 10 & 82 \\
\hline 0 & ENAH & 12 & 35 & 119 & 2 & 85 \\
\hline 0 & CESUES & 289 & 8 & 7,483 & 39 & 87 \\
\hline 0 & UPSLP & 37 & 4 & 3,893 & 6 & 88 \\
\hline 0 & UPTLAX & 37 & 7 & 2,480 & 6 & 95 \\
\hline
\end{tabular}

* Lugar que ocupa en el conteo de las cien IES con mayor número de artículos de 1991 a 2011.

PTC = Profesores de Tiempo Completo. SNI = Investigadores adscritos al Sistema Nacional de Investigadores. pnpc $=$ posgrados ofertados que pertenecen al Programa Nacional de Posgrados de Calidad. MATRÍCULA = número de estudiantes inscritos en las IES en todos los niveles educativos. PROG ACAD = Programas académicos ofertados por la IES.

Fuente: elaboración propia.

Adicionalmente, en el Cuadro 5 se aprecia que la UDG es la segunda IES con mayor número de posgrados de calidad (81 pnpc; 92,451 alumnos; 3,087 PTC y 662 SNI), mientras que el tercer lugar lo ocupa el IPN (73 pnpc; 100,452 alumnos; 4,901 PTC y 662 SNI). Un dato importante derivado de nuestros resultados es que siete de las diez IES con mayor cantidad de artículos publicados corresponden a las instituciones que cuentan con más pnpc; mientras que las universidades que carecen de éstos se ubican en los últimos lugares de la clasificación mencionada.

Finalmente, en el Cuadro 6 se muestra el nombre, con su respectiva sigla o acrónimo de cada una de las IES analizadas. 
Cuadro 6. Siglas o acrónimos de las IES

\begin{tabular}{|c|c|}
\hline Institución & Sigla/acrónimo \\
\hline Benémerita Universidad Autónoma de Puebla & BUAP \\
\hline Centro de Estudios Superiores del Estado de Sonora & Cesues \\
\hline Centro de Ingenieriay Desarrollo Industrial & Cidesi \\
\hline Centro de Innovacion Aplicada en Tecnologías Competitivas, A.C. & Ciatec \\
\hline Centro de Investigación Científica de Yucatán, A.C. & CICY \\
\hline Centro de Investigación Científica y de Educacion Superior de Ensenada, B.C. & Cicese \\
\hline Centro de Investigación en Alimentación y Desarrollo, A.C. & CIAD \\
\hline Centro de Investigación en Matemáticas, A.C. & Cimat \\
\hline Centro de Investigación en Materiales Avanzados, S.C. & Cimav \\
\hline Centro de Investigación en Química Aplicada & CIQA \\
\hline Centro de Investigación y Asistencia en Tecnología y Diseño del Edo. de Jalisco & Ciatej \\
\hline Centro de Investigación y de Estudios Avanzados del ipn & Cinvestav \\
\hline Centro de Investigación y Desarrollo Tecnológico en Electroquímica, S.C. & Cideteq \\
\hline Centro de Investigación y Docencia Economicas, A.C. & CIDE \\
\hline Centro de Investigación y Estudios Superiores en Antropología Social & CIESAS \\
\hline Centro de Investigaciones Biológicas del Noroeste, S.C. & Cibnor \\
\hline Centro de Investigaciones en Óptica, A.C. & $\mathrm{ClO}$ \\
\hline Centro Nacional de Investigación y Desarrollo Tecnológico & Cenidet \\
\hline Centro de Tecnología Avanzada & Ciateq \\
\hline Colegio de Postgraduados en Ciencias Agricolas & Colpos \\
\hline Colegio Superior Agropecuario del Estado de Guerrero & Csaegro \\
\hline Corporación Mexicana de Investigación en Materiales & Comimsa \\
\hline Direccion General de Educación en Ciencia y Tecnología del Mar & DGECYTM \\
\hline Direccion General de Educación Superior Tecnológica & DGEST \\
\hline El Colegio de la Frontera Norte & Colef \\
\hline El Colegio de la Frontera Sur & Ecosur \\
\hline El Colegio de México & Colmex \\
\hline El Colegio de Michoacán & Colmich \\
\hline Escuela Nacional de Antropología e Historia & ENAH \\
\hline Facultad Latinoamericana de Ciencias Sociales & Flacso \\
\hline Instituto de Ecología & Inecol \\
\hline Instituto de Investigaciones "Dr. José María Luis Mora" & IMORA \\
\hline Instituto Nacional de Astrofísica Óptica y Electrónica & INAOE \\
\hline
\end{tabular}




\begin{tabular}{|c|c|}
\hline Institución & Sigla/acrónimo \\
\hline Instituto Politécnico Nacional & IPN \\
\hline Instituto Potosino de Investigación Científica y Tecnológica & IPICYT \\
\hline Instituto Tecnológico Autónomo de México & ITAM \\
\hline Instituto Tecnológico de Sonora & ITSON \\
\hline Instituto Tecnológico y de Estudios Superiores de Monterrey & ITESM \\
\hline Instituto Tecnológico y de Estudios Superiores de Occidente & ITESO \\
\hline Tecnológico de Estudios Superiores de Ecatepec & TESE \\
\hline Universidad Anáhuac del Norte & UAN \\
\hline Universidad Autónoma Agraria Antonio Narro & UAAAN \\
\hline Universidad Autónoma Benito Juárez de Oaxaca & UABJO \\
\hline Universidad Autónoma Chapingo & UACH \\
\hline Universidad Autónoma de Aguascalientes & UAA \\
\hline Universidad Autónoma de Baja California & UABC \\
\hline Universidad Autónoma de Baja California Sur & UABCS \\
\hline Universidad Autónoma de Campeche & UACAM \\
\hline Universidad Autónoma de Chiapas & UNACH \\
\hline Universidad Autónoma de Chihuahua & UACH \\
\hline Universidad Autónoma de Ciudad Juárez & UACJ \\
\hline Universidad Autónoma de Coahuila & UADEC \\
\hline Universidad Autónoma de Guadalajara & UAG \\
\hline Universidad Autónoma de Guerrero & UAGRO \\
\hline Universidad Autónoma de la Ciudad de México & UACM \\
\hline Universidad Autónoma de Nayarit & UAN \\
\hline Universidad Autónoma de Nuevo León & UANL \\
\hline Universidad Autónoma de Queretaro & UAQ \\
\hline Universidad Autónoma de San Luis Potosí & UASLP \\
\hline Universidad Autónoma de Sinaloa & UAS \\
\hline Universidad Autónoma de Tamaulipas & UAT \\
\hline Universidad Autónoma de Tlaxcala & UATX \\
\hline Universidad Autónoma de Yucatán & UADY \\
\hline Universidad Autónoma de Zacatecas & UAZ \\
\hline Universidad Autónoma del Carmen & Unacar \\
\hline Universidad Autónoma del Estado de Hidalgo & UAEH \\
\hline Universidad Autónoma del Estado de México & UAEMEX \\
\hline Universidad Autónoma del Estado de Morelos & UAEM \\
\hline
\end{tabular}


EL SISTEMA NACIONAL DE INVESTIGADORES EN MÉXICO..

\begin{tabular}{|c|c|}
\hline Institución & Sigla/acrónimo \\
\hline Universidad Autónoma Metropolitana & UAM \\
\hline Universidad de Ciencias y Artes de Chiapas & Unicah \\
\hline Universidad de Colima & UCOL \\
\hline Universidad de Guadalajara & UDG \\
\hline Universidad de Guanajuato & UGTO \\
\hline Universidad de La Ciénega del Estado de Michoacán & UCEM \\
\hline Universidad de Las Américas Puebla & UDLAP \\
\hline Universidad de Monterrey & UDEM \\
\hline Universidad de Occidente & UDO \\
\hline Universidad de Sonora & USON \\
\hline Universidad del Ejército y Fuerza Aérea & UDEFA \\
\hline Universidad del Istmo & UNISTMO \\
\hline Universidad del Mar & UMAR \\
\hline Universidad del Mayab, A.C. & UAM \\
\hline Universidad del Papaloapan & UNPA \\
\hline Universidad del Valle de México & UVM \\
\hline Universidad Iberoamericana & Ibero \\
\hline Universidad Juarez Autónoma de Tabasco & UJAT \\
\hline Universidad Juarez del Estado de Durango & UJED \\
\hline Universidad La Salle, A.C. & USALLE \\
\hline Universidad Marista de Mérida & Marista \\
\hline Universidad Michoacana de San Nicolás de Hidalgo & UMICH \\
\hline Universidad Nacional Autónoma de México & UNAM \\
\hline Universidad Panamericana & UP \\
\hline Universidad Pedagogica Nacional & UPN \\
\hline Universidad Politecnica de Aguascalientes & UPA \\
\hline Universidad Politécnica de Chiapas & UPCHIAPAS \\
\hline Universidad Politécnica de Pachuca & UPP \\
\hline Universidad Politécnica de Puebla & UPPUE \\
\hline Universidad Politécnica de San Luis Potosí & UPSLP \\
\hline Universidad Politécnica de Tlaxcala & UPTLAX \\
\hline Universidad Politécnica de Victoria & UPV \\
\hline Universidad Politécnica del Estado de Morelos & UPEMOR \\
\hline Universidad Popular Autónoma del Estado de Puebla & UPAEP \\
\hline Universidad Tecnológica de La Mixteca & UTM \\
\hline
\end{tabular}




\begin{tabular}{|l|l|}
\hline \multicolumn{1}{|c|}{ Institución } & \multicolumn{1}{|c|}{ Sigla/acrónimo } \\
\hline Universidad Tecnológica de Puebla & UTP \\
\hline Universidad Veracruzana & UV \\
\hline
\end{tabular}

Fuente: elaboración propia.

\section{Conclusiones}

El objetivo de este trabajo fue analizar cómo un sistema de estímulos económicos y de reconocimiento a las actividades de investigación en México ha impactado durante 21 años (1991-2011) en la productividad científica de los investigadores adscritos a una IES y, al mismo tiempo, cómo dichas instituciones han sido beneficiadas por ese sistema. Con ese propósito, se elaboró una base de datos con la información de 27,667 investigadores mexicanos que estuvieron adscritos al SNI al menos por un año durante el periodo en cuestión. Así, se obtuvo la información de 122,406 artículos publicados para determinar la cantidad y calidad de la productividad de las IES.

De acuerdo con lo descrito en el portal Execum de la UNAM, existen 177 universidades públicas, 249 institutos tecnológicos y 1835 instituciones de educación privada en México. En este artículo se obtuvo información relevante que indica que, durante dos décadas de estudio, únicamente 156 IES contribuyeron al conocimiento con al menos un artículo publicado en una revista ISI (sólo se mostraron las cien primeras). Los resultados de las clasificaciones muestran que existen muy pocas universidades orientadas a la investigación y que las diez instituciones educativas con mayor cantidad de artículos publicados corresponden a las que cuentan con mayor número de citas bibliográficas. Dentro de estas universidades se encuentran cuatro IES federales (UNAM, Cinvestav, IPN y UAM), cinco estatales (UDG, UANL, UASLP, BUAP y UGTO) y un centro Conacyt (INAOE). Además, debido a que nuestros resultados indican que en dichas IES se cuenta con la más amplia oferta de posgrados registrados al pnpc y se registra el mayor número de ingresos y bajas al SNI, se infiere que dentro de éstas existe un ambiente competitivo y enfocado a mantener la calidad de la investigación realizada.

En México, el SNI representa un mecanismo eficiente para la formación y consolidación de investigadores de excelencia, a partir de la evaluación de la producción académica de sus miembros. Este sistema se ha convertido en un parámetro para la evaluación (clasificación) de las IES a nivel nacional, e indirectamente ha fomentado una aparente competencia por parte de las instituciones en términos de producción científica (artículos, patentes y citas bibliográficas, entre otros), especialmente debido a que las tasas de productividad 
(frecuentemente el número de artículos) son empleadas por instituciones externas para evaluar el desempeño de las IES y con ello obtener algún tipo de financiamiento para actividades vinculadas con la investigación y desarrollo.

"Publicar o salir del sistema" representa un medio para incrementar el salario de los investigadores, debido a que ingresar al sistema representa, en algunos casos, duplicar o triplicar el salario mediante el estímulo económico otorgado por el SNI. En este trabajo se identificó la colaboración como un mecanismo, entre otros, empleado por los investigadores para obtener un estándar de artículos y mantenerse en el sistema, siendo la colaboración entre colegas nacionales la que se presenta de manera más recurrente.

\section{REFERENCIAS}

Alcántara, A. 2009. "Reforma de la educación superior", Revista de la Educación Superior, vol. 38, no. 2: 125-129.

Angell, M. 1986. "Publish or perish: A proposal", Annals of Internal Medicine, vol. 104, 2: 261-262.

Arora, A. y A. Gambardella. 1998. "Impact of nsf support for basic research in economics", Econ. WPA.

Astin, A.W. 1972. "The measured effects of higher education", The Annals of the American Academy of Political and Social Science, vol. 404: 1-20.

Babchuk, N. y A.P. Bates. 1962. "Professor or producer: The two faces of academic man”, Social Forces, vol. 40, no. 4:341-348.

Backes-Gellner, U. y A. Schlinghoff. 2008. "Monetary rewards and faculty behaviour; how economic incentives drive publish or perish", en Southern Management Association Proceedings. St. Pete Beach, Flor.: Southern Management Association (SMA), 725-730.

Bayer, A.E. y J.E. Dutton. 1977. "Career age and research-professional activities of academic scientists: Tests of alternative”, The Journal of Higher Education, vol. 48, no. 3: 259-282.

Behymer, C. 1974. "Institutional and personal correlates of faculty productivity". Ann Arbor: University of Michigan, tesis doctoral.

Behymer, C.E. y R.T. Blackburn. 1975. "Environmental and personal atributes related to faculty productivity", Annual Meeting of the American Educational Research Association (Washington, D.C.).

Chavoya Peña, M.L. 2011. "El impulso a la investigación en las universidades mexicanas", en <www.congresoretosyexpectativas.UDG.mx/Congreso $\% 201 / . . . / m e-$ sa-b_2.p>.

Cole, J.R, y H. Zuckerman. 1984. "The productivity puzzle: Persistence and change in patterns of publication of men and women scientist”, en M.W. Steimkamp y M. 
Maehr, eds., Advances in motivation and achievement, vol. 2. Greenwich: JAI Press.

Cole, S. 1979. "Age and scientific performance", American Journal of Sociology, vol. 84, no. 4: 958-977.

Craswell, J. W. 1986. Measuring faculty research performance. San Francisco: Jossey-Bass.

Crewe, I. 1988. "Reputation, research and reality: The publication records of UK Departments of Politics, 1978-1984", Scientometrics, vol. 14, no. 3: 235-250.

Franklin, N.M. 1989. The community of science in Europe: Preconditions for research effectiveness in European Community countries. Brookfield: Gower.

Galaz, J. F. 1998. "Sobre la clasificación de las instituciones mexicanas de educación superior”, Revista de la Educación Superior, vol. 27, no. 106.

González-Brambila, C. y M. F. Veloso. 2007. "The determinants of research output and impact: A study of Mexican researchers", Research Policy, vol. 36, no. 7: 10351051.

Grediaga Kuri, R. 2006. "Las políticas hacia los académicos en las últimas décadas. Cambios en la regulación de las trayectorias y el sistema de reconocimiento y recompensas de la profesión académica en México”, CPU-e. Revista de Investigación Educativa, vol. 2 (enero-junio): 1-72.

Hadjinicola, G.C. y A.C. Soteriou. 2006. "Factors affecting research productivity of production and operations management groups: An empirical study", Journal of Applied Mathematics and Decision Sciences, vol. 2006. DOI: <http://dx.doi. org/10.1155/JAMDS/2006/96542>.

Ibarrola, M. 1992. "Ecología de la academia en México y Europa occidental”, Avance y Perspectiva, vol. 11 (julio-agosto).

Jacobson, R.L. 1992. "Colleges face new pressure to increase faculty productivity", Chronicle of Higher Education, vol. 38, no. 32: 16-18.

Jordan, M.J., M. Meador y S.J. Walters. 1988. "Effects of department size and organization on the research productivity of academic economists", Economics of Education Review, vol. 7, no. 2: 252-255.

Kotrlik, J.W., J.E. Bartlett, C.C. Higgins y H.A. Williams. 2002. "Factors associated with research productivity of Agricultural Education Faculty”, Journal of Agricultural Education, vol. 43, no. 3: 1-10.

Kyvik, S. 1990. "Age and scientific productivity. Differences", Higher Education, vol. 19, no. 1:37-55.

Lehman, H.L. 1953. Age and achievement. Princeton: Princeton University Press.

Maldonado, A.M. 2010. "Salarios de académicos de instituciones de educación superior en México comparado con treinta países”, XI Congreso Nacional de Investigación Educativa, en <http://www.comie.org.mx/congreso/memoriaelectronica/v11/docs/area_16/2395.pdf>. 
Melguizo, T. y H.M. Strober. 2007. "Faculty salaries and the maximization of prestige”, Research in Higher Education, vol. 48, no. 6: 633-668.

Nature. 2010. "Publish or perish", Nature, vol. 467, no. 7313 (septiembre): 252. doi: $<10.1038 / 467252 \mathrm{a}>$.

OECD. 2002. Steering and funding of research institutions. governance of science systems: New challenges. París: OECD.

Pelz, D.C. y F.M. Andrews. 1966. Scientists in organizations: productive climates for research and development. Oxford: John Wiley and Sons.

Pfeffer, J. y N. Langton. 1993. "The effect of wage dispersion on satisfaction, productivity, and working collaboratively: Evidence from college and university faculty", Administrative Science Quarterly, vol. 38, no. 3: 382-407.

Santiago Rodríguez, F. 2006. "Valoración del Sistema Nacional de Investigadores", en Diagnostico de la política cientifica, tecnológica y de fomento a la innovación en México (2000-2006). México: Foro Consultivo Científico y Tecnológico.

Stack, S. 2004. "Gender, children and research productivity", Research in Higher Education, vol. 45, no. 8: 891-920.

Stephen, C. y J. R. Cole. 1967. "Scientific output and recognition: A study in the operation of the reward system in science", American Sociological Review, vol. 32, no. 3: 377-390.

Vega y León, Salvador. 2003. Sistema Nacional de Investigadores. Retos y perspectivas de la ciencia en México. México: UAM.

\section{Para citar este texto:}

Rodríguez Miramontes, Jorge; González Brambila, Claudia N.; Maqueda Rodríguez, Gabriela. 2017. "El Sistema Nacional de Investigadores en México: 20 años de producción científica en las instituciones de educación superior (1991-2011)". Investigación Bibliotecológica: Archivonomía, Bibliotecología e Información (Número Especial de Bibliometría): 187-219.

http://dx.doi.org/10.22201/iibi.24488321xe.2017.nesp1.57890 